\title{
Nonadherence in difficult asthma - facts, myths, and a time to act
}

\author{
This article was published in the following Dove Press journal: \\ Patient Preference and Adherence \\ I8 April 2013 \\ Number of times this article has been viewed
}

\section{John T Lindsay \\ Liam G Heaney}

Centre for Infection and Immunity, Queens University Belfast, Belfast, UK
Correspondence: Liam Heaney

Centre for Infection and Immunity,

Queen's University Belfast, Level 8,

Belfast City Hospital, Lisburn Road,

Belfast, Northern Ireland, UK BT9 7AB

$\mathrm{Tel}+442895047964$

Fax +442890263879

Email I.heaney@qub.ac.uk
Abstract: Nonadherence to prescribed treatment is an important cause of difficult asthma. Rates of nonadherence amongst asthmatic patients have been shown to range between $30 \%$ and $70 \%$. This is associated with poor health care outcomes and increased health care costs. There is no such thing as a "typical" nonadherent patient. The reasons driving nonadherence are multifactorial. Furthermore, adherence is a variable behavior and not a trait characteristic. Adherence rates can vary between the same individual across treatments for different conditions. There is no consistent link between socioeconomic status and nonadherence, and although some studies have shown that nonadherence is more common amongst females, this is not a universal finding. The commonly held perception that better adherence is driven by greater disease severity has been demonstrated to not be the case, in both pediatric and adult patients. Identification of nonadherence is the first step. If adherence is not checked, it is likely that poor adherence will be labeled as refractory disease. Failure to identify poor adherence may lead to inappropriate escalation of therapy, including the potential introduction of complex biological therapies. Surrogate measures, such as prescription counting, are not infallible. Nonadherence can be difficult to identify in clinical practice, and a systematic approach using a variety of tools is required. Nonadherence can be successfully addressed. Therefore, assessment of adherence is of paramount importance in difficult asthma management, in order to reduce exacerbations and steroid-related side effects as well as hospital and intensive care admissions, health care cost, and inappropriate treatment escalation. In this paper, we present an overview of the literature surrounding nonadherence in difficult asthma. We explore the facts and myths surrounding the factors driving nonadherence as well as how it can be identified and addressed.

Keywords: concordance, compliance, inhaled corticosteroids, drug monitoring

\section{Introduction and objectives}

Asthma is estimated to affect 300 million people worldwide and is a major preventable cause of morbidity and mortality. ${ }^{1,2}$ Approximately $5 \%-10 \%$ of asthmatic patients suffer from "difficult asthma," which can be defined as persistent symptoms and/or frequent exacerbations despite prescription for maximal recommended inhaled corticosteroid (ICS) treatment in addition to other maintenance therapies ${ }^{3}$ (ie, prescribed treatment at Global Initiative For Asthma Step 4/5). ${ }^{4}$ One of the causes of difficult asthma is suboptimal adherence to prescribed medication, principally ICS treatment.

In this paper, we present an overview of the literature on nonadherence in difficult asthma. Our objectives are to review the problem of nonadherence in terms of its prevalence and demographic associations, methods of identification and lastly, the ways in which it can be addressed in clinical practice. We will examine the facts and myths 
surrounding the condition and underline the importance of timely identification.

\section{Definitions}

The World Health Organization (WHO) defines adherence as "the extent to which a person's behavior [...] corresponds with agreed recommendations from a health care provider." "Adherence," as a term, has largely superseded "compliance," which was felt to have a paternalistic connotation, ie, an implied patient subservience in the medication-prescription process. "Adherence" acknowledges the patient's right to choose whether or not to follow these recommendations. Another term that is often used is "concordance," which is a more complex idea incorporating the relationship between doctor and patient, and the extent to which both parties agree on the recommendations; it implies shared decision making in the management of the patient's condition. ${ }^{6}$

Nonadherence is prevalent in all chronic diseases, and, in 1979, a review of over 500 studies identified adherence to be approximately 50\% in different conditions where long-term medication was prescribed, with a range of $0 \%$ to $100 \%{ }^{7}$

\section{Nonadherence to inhaled corticosteroid therapy in asthma}

Rates of nonadherence amongst asthmatic patients (to all medications, including theophylline as measured by serum levels) were shown to range between $30 \%$ and $70 \%$ in a 1997 review; ${ }^{8}$ this is apparent irrespective of how adherence is measured, be it the percentage of prescribed medication taken, serum theophylline levels, days of medication adherence, or percentages of patients who are identified to be nonadherent to medication. ${ }^{8}$

The problem of suboptimal adherence is particularly obvious for ICS even with short-term use. For example, in a small study involving 20 asthmatic participants, after a 5-week study employing electronic monitoring of inhaler use, only $40 \%$ of patients were found to be adherent. ${ }^{9}$ Another study, which looked at the effect of direct clinician-to-patient feedback on ICS use, found an overall baseline adherence to ICS of approximately $56 \% .^{10}$

Nonadherence to ICS is also associated with a poor health care outcome. ${ }^{11}$ A large study looking at 12,636 patients attending the emergency department with an asthma exacerbation revealed that on the basis of pharmacy records, $25.1 \%$ of patients had had ICS dispensed during the 12 months prior compared with $53.5 \%$ who had had a short-acting beta- 2 agonist (SABA) dispensed. ${ }^{12}$ This would indicate an apparent preference of reliever medication over maintenance in this group of asthmatics who had had a severe exacerbation. Two large Canadian case-control studies have looked at the effects of regular ICS use on hospitalization for asthma ${ }^{13}$ and on mortality, ${ }^{14}$ using prescription filling as a surrogate measure of medication use. Both studies drew data from a large health database with precise prescription data. They identified 30,569 asthmatics aged between 5 and 44 years who had received three or more antiasthmatic medication prescriptions in a 1-year period between 1975 and 1991, and who had been followed up for at least 1 year. They looked at ICS prescriptions that were dispensed in the year prior to an admission to hospital. Regular use was defined as at least one ICS inhaler being dispensed per quarter (ie, 3-month period). Irregular use was defined as any other pattern. They found the overall rate of admission to hospital on account of asthma was 42.4 per 1000 asthmatic patients per year (with the rate of first asthma-related hospitalization of 17.2 per 1000 patients per year). Regular use of ICS, as described above, was found to lead to an overall reduction in rate of hospital admission of 34\%. ${ }^{13}$

For the mortality study, ${ }^{14}$ the same standard of regular ICS use was employed. There were 562 deaths in total in the 30,569-strong cohort. Seventy-seven of the deaths were felt to be due to asthma, and there were full data on 66 of these; these cases were matched with control patients from the database with similar duration and severity of disease who did not die. The mean number of ICS inhalers picked up in the 12 months prior to death was 1.18 , compared with 1.57 inhalers for the control group ( $93 \%$ of the inhalers were low-dose beclomethasone). Using a continuous dose-response analysis, the authors calculated a reduction of $21 \%$ in the rate of death from asthma with every additional ICS canister used in the preceding 12 months. They concluded from these data that regular use of low-dose ICS is associated with a reduction in asthma-related mortality at population level. ${ }^{14}$

\section{The "typical" nonadherent patient}

There is a perception that the "typical" nonadherent patient can be easily identified in the clinic - eg, the patient who engages in high-risk behaviors such as smoking, who has a chaotic lifestyle, or who has some other obvious identifiable reason for poor adherence to medication. However, whilst these issues may be relevant in some cases, there is no such thing as a "typical" nonadherent patient, and one has to consider many factors. It is useful to think of nonadherence as falling into two broad categories - nonintentional and intentional - although these should not be thought of as mutually exclusive. ${ }^{15}$ Nonintentional nonadherence occurs when the patient wants to take the medication but is unable 
to as a result of practical barriers - eg, the patient simply forgets to take it, misunderstands or forgets the instructions given by the caregiver, cannot take it (due to poor inhaler technique), can't afford the medication, etc. Intentional nonadherence occurs when the patient decides not to follow treatment recommendations. The reasons for this pattern of behavior are more complex and include denial, medication and disease beliefs, and secondary gain. Barriers, therefore, in the nonintentional and intentional paradigms of nonadherence are practical and perceptual, respectively. Practical barriers can largely be solved by practical solutions. With perceptual barriers, however, a different approach is required.

\section{Demographic associations with poor adherence}

There is no consistent link between socioeconomic status and nonadherence. ${ }^{15}$ A meta-analysis looking at factors associated with adherence did reveal, however, that there was a positive correlation between level of adherence and measures of income, but not with socioeconomic status generally. ${ }^{16}$ Some studies have reported that nonadherence is more common amongst females. A Brazilian study looking at 131 moderate-to-severe asthmatics given free-of-charge ICS/long-acting beta-2 agonist (LABA) combination inhalers for 3 months found that (on the basis of the amount used from each inhaler) being male was protective against nonadherence (where adherence was defined as using more than $85 \%$ of the medication); however, this association was not seen in a multivariate analysis that took into account the other dependent variables, such as race, marital status, smoking status, and asthma severity. Interestingly, in this model, the only consistent association was a higher rate of adherence amongst patients with a greater severity of asthma. ${ }^{17}$

Another study carried out in Detroit, MI, USA examined adherence differences between white patients and African-American patients, and examined data from prescription filling and medical records. ${ }^{18}$ Amongst white patients, female adherence was significantly lower than in men. They also identified poorer adherence in AfricanAmerican patients compared with white patients. AfricanAmerican patients also had a lower median household income and a higher index crime rate in their area of residence and were more likely to live in an inner-city location. Amongst African-American patients, ICS adherence was found to be negatively correlated with local crime rate. ${ }^{18}$ In a related study by the same authors of a different cohort (where adherence was classified as $\geq 80 \%$ prescription filling), they again identified female sex along with younger age and African-American race to be associated with nonadherence. ${ }^{19}$

However the association with female sex is not a universal finding. A postal survey carried out in Portland, OR, USA amongst a sample of adults with persistent asthma, which was specifically designed to assess sex differences in various aspects of the condition, found that women were more likely to regularly monitor their condition via peak flow, to attend a regular clinician for their asthma, and to have a written management plan. ${ }^{20}$ A Swedish study used prescription refilling to assess adherence to ICS, including combination (ICS with LABA) inhalers as well as anticholinergic inhalers, in patients aged 60 years or over with asthma or chronic obstructive pulmonary disease (COPD) (the analysis was done for both diseases together). ${ }^{21}$ This study also failed to notice any sex association with poor adherence. For ICS, it was noted that $59 \%$ of patients were "underadherent" (ie, less than $80 \%$ of prescriptions filled), while another $12 \%$ were found to be "overadherent" (more than $120 \%$ of prescriptions filled), with more oversupply noted with combination inhalers. ${ }^{21}$

A follow-up study to both the European Community Respiratory Health Survey I and II, which assessed how patients' self-reported adherence to asthma treatment had changed since their involvement in the previous surveys, revealed no significant differences between the sexes in terms of either increased adherence or persistence of adherence. ${ }^{22}$ This global study did note that while adherence remained low worldwide, it did increase in continental and extra-European areas. The study also noted that the best predictors of increased or persistent adherence were regular appointments with a specialist and positive beliefs about the medication.

Mood disorder may affect adherence with medication. A positive association has been demonstrated between depressive symptoms and poor adherence (less than $50 \%$ ) to ICS and oral corticosteroid treatment in the 2 weeks after discharge from hospital following an asthma-related admission. ${ }^{23}$

It is also important to note that adherence rates can vary within the same individual across treatments for different conditions. A study carried out via online surveys in the USA looked at cost-related medication underuse for chronic illnesses. ${ }^{24}$ This study demonstrated that large numbers of adults with chronic illnesses had taken less medication than prescribed, specifically because of the cost. Furthermore, participants were noted not to be cutting back uniformly on treatments for different conditions. ${ }^{24}$ Thus, there is no "typical" nonadherent patient. Adherence is a variable behavior and not a trait characteristic, and in fact, most people will be nonadherent some of the time. ${ }^{15}$ 


\section{Adherence in clinical trials}

In a 2 -year-long Norwegian pediatric study, it was noted that there was relatively high adherence at the beginning of the trial period, but that this fell over subsequent months. ${ }^{25}$ This study calculated adherence on the basis of doses used from inhaler devices that were returned; participants were 7- to 16-year-old mild asthmatics and were not told that their adherence was being monitored. This implies that adherence will fall even in the well-monitored clinical trial scenario, making it essential that adherence is always measured in such studies. This study noted that the 7- to 9-year-old group was consistently more adherent than the 10- to 16-year-old group, and significantly so for the first 3 months, which may reflect greater parental influence in younger children. Also, they noted that during the last 6 months of the study, patients on placebo had significantly lower adherence than those receiving ICS, which may reflect the identification of a therapeutic benefit of the ICS. ${ }^{25}$

In a large adult study - the Gaining Optimal Asthma Control Study - ICS and inhaled ICS/LABA combination therapy (ICT) were compared, using a dose escalation strategy, to improve asthma control. ${ }^{26}$ Despite monitoring in the trial, $11 \%$ of patients used less than $80 \%$ of their medication, based on the dose counter on the inhaler device.

\section{Nonadherence and disease severity}

A commonly held perception is that more severe disease, more disease-related morbidity will drive better adherence, and this is particularly the case with a medication like ICS in asthma, which has good clinical efficacy. However, a study at a regional tertiary referral difficult asthma service in Northern Ireland revealed that $35 \%$ of new referrals were filling $50 \%$ or less of prescriptions for ICT. ${ }^{27}$ Consistent with other studies in severe and difficult-to-control asthma, the majority (62\% of the 182 consecutive referrals) were female, and female patients were significantly more likely to be nonadherent to ICT $-42 \%$ female versus $23 \%$ male. Importantly, in this study, the prescription filling as a surrogate of poor adherence was validated using a patient concordance interview. Patients filling $50 \%$ or less of their ICT prescriptions were also more likely to have had three or more hospital admissions in the preceding 12 months. Furthermore, they were more likely to be using home nebulizers, and to use a significantly greater amount of nebulized short-acting beta-2 agonist (SABA) medication. $^{27}$

Similar results were found at another specialist UK difficult asthma service in Leicester. ${ }^{28}$ Using prescription records, suboptimal adherence to ICS and ICT (defined as $<80 \%$ prescriptions filled) was found in 75 of 115 patients $(65.2 \%)$ - of note, in the Belfast study, $<80 \%$ prescription filling was found in $64 \%$ of patients. ${ }^{29}$ Again, poorer asthma control and increased health care utilization was noted. Patients with poor adherence to ICS were found to have a significantly lower postbronchodilator 1-second forced expiratory volume $\left(\mathrm{FEV}_{1}\right)$ (percentage predicted mean $75.5 \%$ vs $84.3 \%$ ) and higher sputum eosinophil count (mean $4.6 \%$ vs $2.3 \%$ ) than those with adequate ICS adherence. ${ }^{28}$ Also, patients with poor prescription filling were more likely to have been admitted to an intensive care unit for ventilation on account of their asthma $(19.2 \%$ vs $2.6 \%) .{ }^{30}$

The finding of poor adherence associated with difficult-tocontrol asthma is also seen in children. In a pediatric study of referrals to a tertiary referral centre in London, 71 children who had persistent symptoms and frequent exacerbations despite treatment of at least step 4 had a nurse-led home visit as part of a multidisciplinary assessment. ${ }^{31}$ Adherence was addressed as part of this visit, which included an examination of the patient's medication usage and inhaler technique. Only $43 \%$ of patients had filled more than $80 \%$ of prescriptions for their asthma medication issued, and $30 \%$ of patients had filled less than $50 \%$ asthma prescriptions issued. Poor symptom control found in $48 \%$ of the children under study was felt to be contributed to by poor adherence, unsuitable device, and poor inhaler technique. ${ }^{31}$

\section{Identifying nonadherence in asthma}

Surrogate measures of adherence, such as prescription counting and inhaler weighing/dose counting, are not infallible. Patients may pick up their inhalers, but not use them. Also, prescription records give a percentage of the amount of inhalers that should have been used over a certain time period - ie, 6-12 months. This gives information about long-term use but does not acknowledge short-term changes in adherence behavior. Inhaler weighing and dose counting can be confounded by "test doses" or "dumping," in which the medication is simply discharged several times by the patient to bring ostensible usage up to a more "satisfactory" level. ${ }^{32}$

More objective measures for monitoring inhaled medication are available, such as electronic monitoring, but these are expensive for routine clinical use and prone to the Hawthorne effect, whereby patients improve their adherence transiently when they know that it is being monitored.

Physician assessment, patient diaries, and self-reporting have been shown to give an overestimation of compliance when compared with objective electronic measures. ${ }^{11,33-35}$ 
The accuracy of self-reporting is likely to be dependent on the reason for nonadherence and the setting of the study - eg, an anonymous questionnaire is more likely to be precise and less open to overestimation than a one-to-one interview at clinic.

In the Northern Ireland study, 45 of 51 (88\%) patients who were found to be filling $50 \%$ or fewer ICT prescriptions admitted variability in taking their combination treatment, in accordance with the degree of nonadherence identified on prescription records at a subsequent clinical interview. ${ }^{27}$ Of the six patients who continued to deny nonadherence at the interview, three were found to be nonadherent to prednisolone or theophylline, based on serum assays (despite also reporting to be taking these medications). However, all patients had denied nonadherence prior to the concordance interview with the prescription records, and it was felt that in the absence of the prescription data, a concordance interview would not have been feasible, as the patients would not have felt able to admit and discuss their nonadherence.

A similar picture emerged with oral prednisolone, where $86 \%$ of the patients interviewed who had been found to be nonadherent to oral prednisolone, based on serum prednisolone/cortisol measurements, admitted poor adherence. ${ }^{27}$ This study emphasized the importance and utility of objective markers of nonadherence to facilitate a concordance discussion.

Similar findings were obtained in a study carried out at the Royal Brompton Hospital difficult asthma clinic. ${ }^{36}$ Of 100 newly referred patients who were evaluated, 28 patients were receiving $15 \mathrm{mg}$ or more of maintenance prednisolone. Of these, nine $(32.1 \%)$ were found to be nonadherent on the basis of prednisolone/cortisol blood assays.

We have recently demonstrated that the degree of suppression in fractional exhaled nitric oxide (FeNO) occurring with directly observed inhaled steroid therapy can be used to identify nonadherence to ICS in a difficult asthma population. ${ }^{37}$ This test could be used prior to the introduction to complex biological therapies targeting eosinophilic disease (eg, mepolizumab), to ensure the clinical problem is not due to nonadherence with inhaled steroids. We are currently examining delivery of this test using remote monitoring technologies, and in the specialized difficult asthma setting, this test may have an important role in distinguishing the truly refractory patient requiring treatment escalation from the nonadherent patient.

\section{Do oral treatments or once-a-day inhaler regimes improve adherence?}

A large multicenter study, in which 1037 mild-to-moderate asthmatics were analyzed, looked at once-daily versus twice-daily dosing of ICS treatment. ${ }^{38}$ Adherence was measured by dose counter on the inhaler and by patient self-reporting. The trial was of a short duration (12 weeks), and visits were on a monthly basis. Patients were not told that adherence was being observed. Adherence was found to be significantly higher (using the dose counter) in the oncedaily dosing group (once-daily adherence, 93.3\%; twice daily, $89.5 \%$ ). There were no differences in health care outcomes between the two groups. The adherence rates in both groups were very high, and this may be due to the short duration of the study coupled with the Hawthorne effect. A study of longer duration would be beneficial in order to investigate this further.

A year-long study looked at adherence in economically disadvantaged African-American young people. ${ }^{39}$ Researchers used electronic monitoring to look at the adherence to inhaled fluticasone and oral montelukast in 346 participants. Adherence to montelukast was higher than to fluticasone, but over the course of the 12 months, adherence to both medications fell drastically (at the end of the year, participants were taking $23 \%$ of prescribed doses of ICS and $31 \%$ of montelukast). They noted no relationship between adherence and clinical outcome in the overall population. In subjects with "worse asthma," as defined by decreased lung function and increased use of rescue medication, there was better clinical response with better inhaled steroid adherence. In addition, during an open-label extension to the study, with a simplified regime, adherence was worse with both medications. ${ }^{39}$

\section{When identified, is nonadherence easy to address in clinical practice?}

A Cochrane review looked at interventions for enhancing adherence to medication. ${ }^{40}$ They looked at 13 asthma intervention studies in different clinical settings, eg, postadmission and primary/secondary care, with the "intervention" usually being a management plan. These studies involved mixed populations, eg, asthma and COPD, asthma, and rhinitis. Six of these reported adherence improvement using different measures, eg, patient self-report and/or physician estimate. Only two studies used prescription records. Three studies showed changes in a health care outcome, eg, improved peak flow or use of reliever. They concluded that the data was "surprisingly weak" and that "increasing effectiveness of adherence interventions" would have a "greater impact than improvement in specific medical treatments." ${ }^{\prime \prime}$

We have shown in a recent study that 31 of 83 patients (37\%) identified as nonadherent using prescription records 
( $\leq 50 \%$ filling) became adherent following a concordance interview, ${ }^{41}$ which involved conveying the fact to patients that they were nonadherent and agreeing on a treatment plan to improve adherence. This newly adherent group was predominantly female (68\%), and $39 \%$ had been tertiary referrals. Twenty-seven patients $(90 \%)$ remained adherent at 12 to 18 months following the concordance discussion. Improved adherence was again associated with improved outcomes - prescribed daily dose of ICS and number of rescue courses of prednisolone were significantly reduced. $\mathrm{FEV}_{1}$ was significantly improved and there were significantly less hospital admissions on account of asthma. We also carried out a small randomized controlled trial $(n=18)$ of 1 -year duration in which nonadherent patients were randomly allocated to receive either a specially designed menu-driven behavioral intervention in addition to the usual care from the difficult asthma service, or the usual care only. ${ }^{41}$ The primary outcome of "\% combination ICT inhalers filled" was significantly improved $(37.6 \%$ to $61.9 \%$ in the intervention group vs $31.7 \%$ to $28.8 \%$ in the control group). The number needed to treat was found to be three.

\section{The implications of nonadherence in difficult asthma}

Nonadherence in asthma, particularly those with more difficultto-control asthma, is associated with poor asthma outcome. ${ }^{27,28,31}$ It also leads to increased health care cost. ${ }^{8,42-44}$

Nonadherence in difficult asthma can also lead to problems in identifying and understanding refractory asthma. All definitions of refractory or severe asthma are based on both a failure to respond to high dose asthma therapy and the assumption that the patient is taking this therapy adequately. If adherence is not checked, then it is likely that poor adherence will be labeled as refractory disease. Furthermore, failure to identify poor adherence will also lead to inappropriate escalation of therapy, including the potential introduction of complex biological therapies. The current National Institute for Clinical Excellence guidance on the use of omalizumab indicate that it should be used as an "add-on to optimised standard therapy," which is defined as

a full trial of, [...] and documented compliance with inhaled high-dose corticosteroids, long-acting beta- 2 agonists, leukotriene receptor antagonists, theophyllines, oral corticosteroids, and smoking cessation if clinically appropriate. $^{45}$
It does not indicate how this "compliance" should be checked. The guidance recommends that treatment with omalizumab be considered in

people aged 6 years and older who need continuous or frequent treatment with oral corticosteroids (defined as 4 or more courses in the previous year). ${ }^{45}$

The problem remains that it may be simple nonadherence to combination inhaled therapy that leads to patients being prescribed maintenance or rescue treatment with oral corticosteroids and that expensive monoclonal antibody therapies may not be necessary to achieve disease control. This is a situation in which the target population identified by the National Institute for Clinical Excellence does not always align with clinical appropriateness if nonadherence is driving the "continuous or frequent treatment with oral corticosteroids." 45

Nonadherence also poses problems with phenotypespecific prescribing in refractory asthma. The novel anti-Interleukin (IL)-5 monoclonal antibody treatment mepolizumab has been shown to reduce the frequency of exacerbations in refractory eosinophilic asthma. ${ }^{46,47}$ However, nonadherence has been shown to be associated with significantly higher sputum eosinophils. ${ }^{28}$ Therefore, once again it may be that it is simply a question of identifying and addressing nonadherence rather than escalating to an expensive complex therapy. We believe tests such as the FeNO-suppression test, allied with other direct and surrogate measures of nonadherence should be examined prior to commencing complex biologic therapies.

\section{Conclusion}

Nonadherence is very common across all severities of asthma and is a common reason for "difficult asthma" in adults and children. It is difficult to identify in routine clinical practice, requiring a systematic approach using a variety of tools. Biomarkers of adherence or of steroid exposure should be used to prevent inappropriate escalation to complex therapies in difficult asthma. When nonadherence is identified, it can be successfully targeted. Furthermore, it is important to remember that the definitions of severe asthma and the guidelines for phenotype-specific management are all predicated on the assumption of treatment adherence.

\section{Authors' contributions}

LGH: conception and design of paper; JTL: drafting paper; JTL/LGH: review and approval. 


\section{Disclosure}

LGH has received grant funding from Medimmune, Novartis UK, Genentech Inc, and Glaxo Smith Kline, and has taken part in advisory boards and given lectures at meetings supported by Glaxo Smith Kline, Merck Sharpe and Dohme, Nycomed, Novartis, and AstraZeneca. He has received support funding to attend international respiratory meetings (AstraZeneca, Chiesi, Novartis, and Glaxo Smith Kline) and has taken part in asthma clinical trials (GSK and Genentech) for which his institution was remunerated. None of these activities have any direct relationship to the content of this manuscript. JTL reports no conflicts of interest in this work.

\section{References}

1. Masoli M, Fabian D, Holt S, Beasley R; the Global Initiative for Asthma (GINA) Program. The global burden of asthma: executive summary of the GINA Dissemination Committee Report. Allergy. 2004;59(5): 469-478.

2. Asthma [webpage on the Internet]. Geneva: World Health Organization; 2012. Available from: http://www.who.int/topics/asthma/en/. Accessed October 19, 2012.

3. Barnes PJ, Woolcock AJ. Difficult asthma. Eur Respir J. 1998;12(5): 1209-1218.

4. Global Initiative for Asthma. Global Strategy for Asthma Management and Prevention; Global Initiative for Asthma; 2010. Available from: http://www.ginasthma.org/pdf/GINA_Report_2010.pdf. Accessed October 20, 2012.

5. World Health Organization. Adherence to Long-Term Therapies: Evidence for Action. Geneva: World Health Organization; 2003. Available from: http://whqlibdoc.who.int/publications/2003/9241545992.pdf. Accessed October 20, 2012.

6. Royal Pharmaceutical Society of Great Britain. From Compliance to Concordance: Achieving Shared Goals in Medicine Taking. London: Royal Pharmaceutical Society of Great Britain and Merck Sharp and Dohme; 1997.

7. Sackett DL, Snow JC. The magnitude of adherence and non-adherence. In: Haynes RB, Taylor DW, Sackett DL, editors. Compliance in Health Care. Baltimore: Johns Hopkins University Press; 1979: 11-22.

8. Bender B, Milgrom H, Rand C. Nonadherence in asthmatic patients: is there a solution to the problem? Ann Allergy Asthma Immunol. 1997; 79(3):177-186.

9. Chmelik F, Doughty A. Objective measurements of compliance in asthma treatment. Ann Allergy. 1994;73(6):527-532.

10. Onyirimba F, Apter A, Reisine S, et al. Direct clinician-to-patient feedback discussion of inhaled steroid use: its effect on adherence. Ann Allergy Asthma Immunol. 2003;90(4):411-415.

11. Bender B, Milgrom H, Apter A. Adherence intervention research: what have we learned and what do we do next? J Allergy Clin Immunol. 2003;112(3):489-494.

12. Stempel DA, Roberts CS, Stanford RH. Treatment patterns in the months prior to and after asthma-related emergency department visit. Chest. 2004;126(1):75-80.

13. Suissa S, Ernst P, Kezouh A. Regular use of inhaled corticosteroids and the long term prevention of hospitalisation for asthma. Thorax 2002;57(10):880-884.

14. Suissa S, Ernst P, Benayoun S, Baltzan M, Cai B. Low-dose inhaled corticosteroids and the prevention of death from asthma. NEngl J Med. 2000;343(5):332-336.

15. Horne R. Compliance, adherence, and concordance: implications for asthma treatment. Chest. 2006;130(Suppl 1):S65-S72.
16. DiMatteo MR. Variations in patient's adherence to medical recommendations: a quantitative review of 50 years of research. Med Care. 2004; 42(3):200-209.

17. Chatkin JM, Cavelet-Blanco D, Scaglia NC, Tonietto RG, Wagner MB, Fritscher CC. Compliance with maintenance treatment of asthma (ADERE study). J Bras Pneumol. 2006;32(4):277-283.

18. Williams LK, Joseph CL, Peterson EL, et al. Race-ethnicity, crime, and other factors associated with adherence to inhaled corticosteroids. J Allergy Clin Immunol. 2007;119(1):168-175.

19. Williams LK, Joseph CL, Peterson EL, et al. Patients with asthma who do not fill their inhaled corticosteroids: a study of primary nonadherence. J Allergy Clin Immunol. 2007;120(5):1153-1159.

20. Naleway AL, Vollmer WM, Frazier EA, O’Connor E, Magid DJ. Gender differences in asthma management and quality of life. JAsthma. 2006;43(7):549-552.

21. Krigsman K, Moen J, Nilsson JLG, Ring L. Refill adherence by the elderly for asthma/chronic obstructive pulmonary disease drugs dispensed over a 10-year period. J Clin Pharm Ther. 2007;32(6): 603-611.

22. Corsico AG, Cazzoletti L, de Marco R, et al. Factors affecting adherence to asthma treatment in an international cohort of young and middle-aged adults. Respir Med. 2007;101(6):1363-1367.

23. Smith A, Krishnan JA, Bilderback A, Riekert K, Rand CS, Bartlett SJ. Depressive symptoms and adherence to asthma therapy after hospital discharge. Chest. 2006;130(4):1034-1038.

24. Piette JD, Heisler M, Wagner TH. Cost-related medication underuse among chronically ill adults : the treatments people forgo, how often, and who is at risk. Am J Public Health. 2004;94(10):1782-1787.

25. Jónasson G, Carlson KH, Mowinckel P. Asthma drug adherence in a long term clinical trial. Arch Dis Child. 2000;83(4):330-333.

26. Bateman ED, Boushey HA, Bousquet J, et al; the GOAL Investigators Group. Can guideline-defined asthma control be achieved? The Gaining Optimal Asthma Control Study. Am J Respir Crit Care Med. 2004;170(8):836-844.

27. Gamble J, Stevenson M, McClean E, Heaney LG. The prevalence of nonadherence in difficult asthma. Am J Respir Crit Care Med. 2009; 180(9):817-822.

28. Murphy A, Proeschal A, Brightling CE, et al. The relationship between clinical outcomes and medication adherence in difficult-to-control asthma. Thorax. 2012;67(8):751-753.

29. Heaney LG, Horne R. Non-adherence in difficult asthma: time to take it seriously. Thorax. 2012;67:268-270.

30. Murphy AC, Proeschal A, Linnett ME, et al. Identifying non-adherence with asthma medication and the relationship to clinical outcomes amongst adults with difficult-to-control asthma [abstract]. Thorax. 2010;65 Suppl 4:A151.

31. Bracken M, Fleming L, Hall P, et al. The importance of nurse-led home visits in the assessment of children with problematic asthma. Arch Dis Child. 2009;94(10):780-784.

32. Rand CS, Wise RA, Nides M, et al. Metered-dose inhaler adherence in a clinical trial. Am Rev Respir Dis. 1992;146(6):1559-1564.

33. Cochrane MG, Bala MV, Downs KE, Mauskopf J, Ben-Joseph RH. Inhaled corticosteroids for asthma therapy. patient compliance, devices, and inhalation technique. Chest. 2000;117(2):542-550.

34. Braunstein GL, Trinquet G, Harper AE. Compliance with nedocromil sodium and a nedocromil sodium/salbutamol combination. Eur Respir J. 1996;9(5):893-898.

35. Berg J, Dunbar-Jacob J, Sereika SM. An evaluation of a self-management program for adults with asthma. Clin Nurs Res. 1997;6(3):225-238.

36. Robinson DS, Campbell DA, Durham SR, Pfeffer J, Barnes PJ, Chung KF; for the Asthma and Allergy Research Group of the National Heart and Lung Institute. Systematic assessment of difficult-to-treat asthma. Eur Respir J. 2003;22(3):478-483.

37. McNicholl DM, Stevenson M, McGarvey LP, Heaney LG. The utility of fractional exhaled nitric oxide suppression in the identification of nonadherence in difficult asthma. Am J Resp Crit Care Med. 2012; 186(11):1102-1108. 
38. Price D, Robertson A, Bullen K, Rand C, Horne R, Staudinger H. Improved adherence with once-daily versus twice-daily dosing of mometasone furoate administered via dry powder inhaler: a randomized open-label study. BMC Pulm Med. 2010;10:1.

39. McNally KA, Rohan J, Schluchter M, et al. Adherence to combined montelukast and fluticasone treatment in economically disadvantaged African American youth with asthma. J Asthma. 2009;46(9): 921-927.

40. Haynes RB, Ackloo E, Sahota N, McDonald HP, Yao X. Interventions for enhancing medication adherence. Cochrane Database Syst Rev. 2008;2:CD000011.

41. Gamble J, Stevenson M, Heaney LG. A study of a multi-level intervention to improve non-adherence in difficult to control asthma. Respir Med. 2011;105(9):1308-1315.

42. O'Neill C, Gamble J, Lindsay JT, Heaney LG. The impact of nonadherence to inhaled long-acting beta2-adrenoceptor agonist/corticosteroid combination therapy on healthcare costs in difficult-to-control asthma. Pharmaceut Med. 2011;25(6):379-385.
43. Bender BG, Rand C. Medication non-adherence and asthma treatment cost. Curr Opin Allergy Clin Immunol. 2004;4(3):191-195.

44. Tan H, Sarawate C, Singer J, et al. Impact of asthma controller medications on clinical, economic, and patient-reported outcomes. Mayo Clin Proc. 2009;84(8):675-684.

45. Asthma (severe, persistent, patients aged 6+, adults)-omalizumab (rev TA133, TA201): final appraisal determination document [webpage on the Internet]. National Institute for Health and Clinical Excellence; 2013. Available from: http:/guidance.nice.org.uk/TA/WaveR/110/FAD/ FinalAppraisalDetermination/pdf/English. Accessed March 25, 2013.

46. Haldar P, Brightling CE, Hargadon B, et al. Mepolizumab and exacerbations of refractory eosinophilic asthma. $N$ Engl J Med. 2009; 360(10):973-984.

47. Pavord ID, Korn S, Howarth P, et al. Mepolizumab for severe eosinophilic asthma (DREAM): a multicentre, double-blind, placebo-controlled trial. Lancet. 2012;380(9842):651-659.

\section{Publish your work in this journal}

Patient Preference and Adherence is an international, peer-reviewed, open access journal focusing on the growing importance of patient preference and adherence throughout the therapeutic continuum. Patient satisfaction, acceptability, quality of life, compliance, persistence and their role in developing new therapeutic modalities and compounds to optimize clinical outcomes for existing disease states are major areas of interest. This journal has been accepted for indexing on PubMed Central. The manuscript management system is completely online and includes a very quick and fair peer-review system. Visit http://www.dovepress.com/ testimonials.php to read real quotes from published authors. 